\title{
Atopic Patients Show Increased Interleukin 4 Plasma Levels but the Degree of Elevation Is Not Sufficient to Upregulate Interleukin-4-Sensitive Genes
}

\author{
Eugenia Marbach-Breitrück ${ }^{\mathrm{a}}$ Andrea Kalledat ${ }^{\mathrm{a}}$ Dagmar Heydeck $^{\mathrm{a}}$ \\ Sabine Stehling ${ }^{a}$ Joachim W. Fluhr ${ }^{b}$ Torsten Zuberbier ${ }^{b}$ Hartmut Kuhn ${ }^{a}$ \\ ${ }^{a}$ Institute of Biochemistry, Charité - Universitätsmedizin Berlin, Freie Universität Berlin, Humboldt-Universität zu \\ Berlin and Berlin Institute of Health, Berlin, Germany; ${ }^{b}$ Department of Dermatology and Allergology, Charité - \\ Universitätsmedizin Berlin, Freie Universität Berlin, Humboldt-Universität zu Berlin and Berlin Institute of Health, \\ Berlin, Germany
}

\section{Keywords}

Atopic diseases · Interleukins · Lipid mediators .

Lipoxygenases · Monocytes

\begin{abstract}
Background: Atopic diseases constitute a major health challenge for industrialized countries, and elevated levels of interleukin 4 (IL-4) frequently characterize these disorders. Previous in vitro analyses have indicated that IL-4 strongly upregulates the expression of IL-4-sensitive genes in human monocytes. Objective: To explore whether similar expression alterations may contribute to the pathomechanisms of atopic diseases in vivo we carried out a small-scale case-control clinical study $(n=43)$, in which we quantified the plasma levels of IgE and IL-4 as well as the expression of selected IL4-sensitive genes in blood leukocytes. Methods: 34 allergic patients suffering from allergic rhinitis $(n=11)$, atopic eczema $(n=11)$ and allergic asthma $(n=12)$ as well as 9 healthy control individuals were recruited. IgE and IL-4 plasma levels were determined by ELISA, and the expression of selected IL-4-sensitive gene products in blood leukocytes was quanti-
\end{abstract}

(c) 2019 S. Karger AG, Basel

\section{KARGER
E-Mail karger@karger.com
www.karger.com/spp \\ KARGER
E-Mail karger@karger.com
www.karger.com/spp}

fied by qRT-PCR. In addition, the fatty acid oxygenase activity of isolated monocytes was measured by RP-HPLC analysis of the arachidonic acid oxygenation products (ex vivo activity assays). Results: We found that plasma levels of IgE and IL-4 were significantly elevated in atopic patients but the degree of elevation was not sufficient to upregulate the expression of the selected IL-4-sensitive genes in circulating leukocytes. Moreover, the arachidonic acid oxygenase activity of blood monocytes was not significantly altered in atopic patients. Conclusion: Our data suggest that the IL-4 plasma levels of atopic patients are not high enough to impact the expression of IL-4-sensitive genes.

(c) 2019 S. Karger AG, Basel

\section{Introduction}

Allergic diseases are among the most common chronic disorders in all industrialized countries [1]. Their incidence and prevalence have continuously been rising in recent years [2], and millions of people around the world are affected [3]. The chronic inflammatory processes as-

Dr. Hartmut Kuhn 
sociated with allergic disorders, as e.g. atopic dermatitis, are even discussed to be linked to psychological comorbidities such as depression [4]. The pathomechanisms of allergic diseases are still unclear [5], and except for antigen avoidance there is no causal therapy. Eicosanoids such as prostaglandins [6] and leukotrienes [7] have been implicated in the pathogenesis of allergic disorders, and inhibitors of leukotriene biosynthesis are currently available for prescription as antiasthmatic medication [8]. Atopic diseases, such as atopic rhinitis and atopic dermatitis [9], involve a more or less severe dysregulation of the adaptive immune response, and frequently IgE blood levels are elevated [10-12]. In severe cases anti-IgE treatment is even used as therapeutic strategy [13]. Interleukin-4 (IL-4) is a Th2 cytokine, which is secreted during atopic flares [14]. In healthy humans IL-4 plasma concentrations vary between 2 and $39 \mathrm{pg} / \mathrm{mL}[11,15-18]$ but in one study even higher concentrations $(>450 \mathrm{pg} / \mathrm{mL})$ were found [16]. For atopic patients conflicting data have been published [11, 16-19]. When human peripheral monocytes are cultured in vitro in the presence of IL-4, the expression of a complex array of genes was altered [20]. The most strongly upregulated gene product (300-fold) was $A L O X 15$ [20] but more recent studies suggested a parallel upregulation of $A L O X 15 B$ expression [21]. Unfortunately, the in vivo relevance of this in vitro effect has never been investigated.

To fill this gap we here addressed the following questions: (i) Are IL-4 plasma levels elevated in atopic patients? (ii) Is the degree of elevation high enough to induce expression of selected IL-4-sensitive genes? (iii) Is there a statistic correlation between the IL- 4 plasma levels and the expression of these genes? (iv) Are the IL-4 levels of atopic patients sufficiently high to upregulate the fatty acid oxygenase activity of blood monocytes?

\section{Materials and Methods}

\section{Study Design, Patients and Healthy Controls}

The study was carried out as 2-phase clinical case-control trial at the Department of Dermatology and Allergology at the Charité - Universitätsmedizin, Berlin. During the first phase circulating total IgE and IL-4 concentrations were determined in the plasma of atopic patients and of corresponding controls. Expression of ALOX isoforms and other IL-4-sensitive genes was quantified in peripheral leukocytes. During the second phase peripheral monocytes were prepared from atopic patients and healthy controls, and their endogenous arachidonic acid oxygenase activities were evaluated. Patients were recruited from the patient pool of the clinic and underwent complete clinical evaluation including physical examination. Basic blood parameters were determined, and a skin prick test was carried out routinely.

Lipoxygenase Expression in Atopy
For the first study cohort, 43 adult subjects (mixed gender) with an average age of 45 years (online suppl. Table S1, for all online suppl. material, see www.karger.com/doi/10.1159/000/499431) were recruited. 34 patients with a clinical atopic disease history (atopic asthma, atopic eczema, atopic rhinitis) were included. When different atopic symptoms were diagnosed in a single individual, this patient was allocated to the disease group with the most prominent symptoms. As respective control group, 9 healthy volunteers (mixed gender) who never experienced atopic episodes were recruited. The second cohort comprised a total of 38 individuals with an average age of 38 years, including 21 patients with a known atopic history and 17 healthy controls. A written informed consent was obtained from each patient. The study was approved by the local ethics committee under the permission No. EA1/052/16.

\section{Blood Withdrawal, RNA Preparation and Reverse}

Transcription

EDTA blood was obtained by venipuncture. For plasma collection blood samples were centrifuged at $4,000 \mathrm{~g}$ at $4^{\circ} \mathrm{C}$ for $10 \mathrm{~min}$. The plasma layers were collected and stored at $-80^{\circ} \mathrm{C}$. RNA preparation from whole blood samples was performed using a QIAamp RNA Blood Mini Kit (Qiagen, Hilden, Germany). cDNA synthesis was performed with $0.5 \mu \mathrm{g}$ of total RNA using the RevertAid ${ }^{\mathrm{TM}}$ Premium First-Strand cDNA Synthesis Kit (Thermo Fischer Scientific, Germany).

\section{Quantification of Plasma Levels of IgE and IL-4}

Total circulating IgE levels were determined in the Central Laboratory Unit of Charité (Labor Berlin Charité Vivantes $\mathrm{GmbH}$ ) using the ImmunoCAP-Test (UNICAP-250). IgE quantification was carried out for 27 individuals since 7 patients had previously received anti-IgE therapy (omalizumab). IL-4 serum levels were quantified employing a highly sensitive ELISA kit (IBL International, Hamburg, Germany), which was used according to the manufacturer's instruction.

\section{Quantitative Real-Time Polymerase Chain Reaction}

To quantify the expression level of $h A L O X 15, h A L O X 15 B$, $h A L O X 12, h A L O X 5$, human monoamine oxidase $\mathrm{A}(h M A O A)$ and fibronectin $(F N)$ quantitative real-time polymerase chain reaction (qRT-PCR) was carried out on a Rotor Gene 3000 device (Corbett Research, Mortlake, Australia) using the SensiMix ${ }^{\mathrm{TM}}$ SYBR PCR Kit (Bioline, Luckenwalde, Germany). For each target gene, specific exon-spanning amplification primers (online suppl. Table S2) were synthesized (BioTez, Berlin, Germany). The expression of target genes was normalized to GAPDH expression. The $n$-fold change differences for each gene were calculated by relating the patient individual expression levels to the mean of gene expression of all samples.

\section{Monocyte Preparation, ALOX Activity Assay and}

High-Performance Liquid Chromatography Analysis

Human peripheral monocytes were prepared from heparinized human blood by density step gradient centrifugation using Ficoll Paque $(\rho=1.077 \mathrm{~g} / \mathrm{mL})$ and adherence to plastic dishes [22]. To quantify their fatty acid oxygenase activity, monocytes $\left(10^{6}\right.$ cells/ $\mathrm{mL}$ PBS) were incubated in vitro with arachidonic acid $(100 \mu \mathrm{M})$ for $15 \mathrm{~min}$ at $37^{\circ} \mathrm{C}$ (assay volume $0.5 \mathrm{~mL}$ ). The hydroperoxy fatty acids were reduced with sodium borohydride, and protein was 


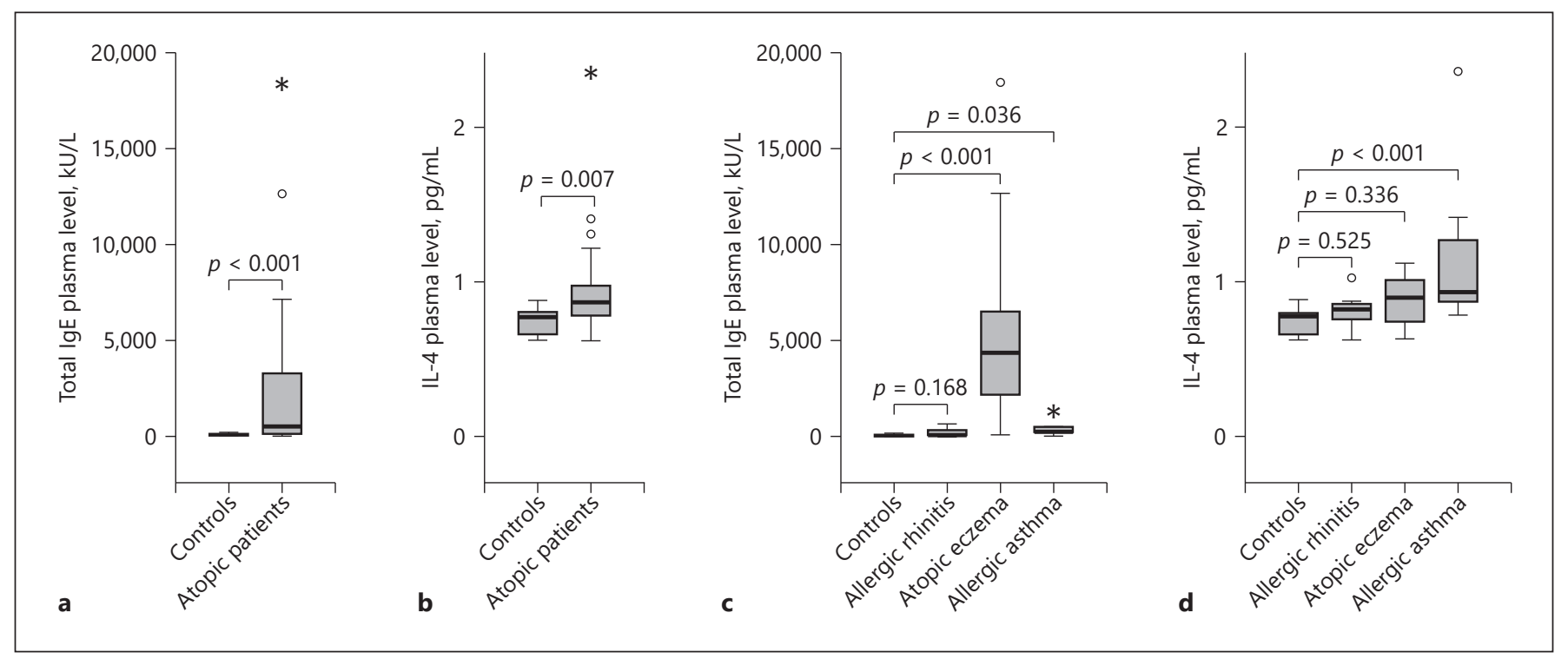

Fig. 1. Concentrations of total IgE and IL- 4 in the blood plasma of healthy human volunteers and patients suffering from atopic diseases. Comparison of total IgE (a) and IL-4 (b) plasma levels of healthy volunteers $(n=9)$ and atopic patients $(n=27$ for IgE and $n=34$ for IL-4, respectively) independent of the pathological entities. Comparison of IgE (c) and IL-4 (d) plasma levels of healthy volunteers $(n=9)$ and atopic patients separated for their pathological entities (allergic rhinitis $n=11$, atopic eczema $n=11$, atopic asthma $n=5$ for IgE and $n=12$ for IL-4, respectively). Statistical evaluation was carried out applying the nonparametric MannWhitney $\mathrm{U}$ test with subsequent Bonferroni adjustment; $p<0.05$ was considered as statistically significant. precipitated with $0.5 \mathrm{~mL}$ ice-cold methanol. Denatured protein was spun down, and aliquots of the clear supernatant were analyzed by reversed-phase high-performance liquid chromatography (RP-HPLC) [22].

\section{Statistical Evaluation of the Experimental Data}

Statistical evaluation of the data was performed using the IBM SPSS Statistics software packages. Since most data were not normally distributed, the nonparametric Mann-Whitney U test was applied (cases vs. controls). When performing multiple statistical comparisons on the same dependent variable, a Bonferroni adjustment was performed. For correlation analysis, the 2-tailed Spearman $\rho$ test was carried out. $p$ values $<0.05$ were considered as statistically significant.

\section{Results}

Plasma Concentrations of IgE and IL-4 Are Elevated in Atopic Patients

When we quantified the IgE and IL-4 plasma concentrations of healthy volunteers and atopic patients, we found that consistent with literature reports [11,1619] the circulating IgE levels (Fig. 1a) were significantly $(p<0.001)$ elevated in atopic patients $(2,550 \pm 833$ vs. $51 \pm 18 \mathrm{kU} / \mathrm{mL})$. A significant increase $(p=0.007)$ was also observed for plasma IL-4 levels (Fig. 1b) (0.94 \pm
0.05 vs. $0.75 \pm 0.03 \mathrm{pg} / \mathrm{mL})$. Next, we separately analyzed the total IgE (Fig. 1c) and IL-4 levels (Fig. 1d) for the different atopic entities and found no significant $(p=0.168)$ elevation of the IgE levels in patients with allergic rhinitis. In contrast, atopic eczema and atopic asthma patients showed significantly augmented $\operatorname{IgE}$ plasma levels ( $p<0.001$ for atopic eczema and $p=0.036$ for allergic asthma). For circulating IL-4 (Fig. 1d), we detected a significant $(p<0.001)$ increase in atopic asthma patients. Atopic eczema $(p=0.525)$ and atopic rhinitis patients $(p=0.336)$ showed no significant alterations.

\section{Expression of IL-4-Sensitive Genes in Blood Leukocytes}

To explore whether the increased IL-4 plasma concentrations in atopic patients induce the expression of selected IL-4-sensitive genes in peripheral leukocytes, we quantified the corresponding mRNAs by qRT-PCR. Here we did not observe significant alterations for $A L O X 15$ but a statistical trend $(p=0.053)$ for increased $A L O X 15 B$ expression (Fig. 2a). No significant differences were observed for ALOX5 and ALOX12.

When we separately analyzed the different disease entities we detected a nonsignificant $(p=0.168)$ 2.57- 


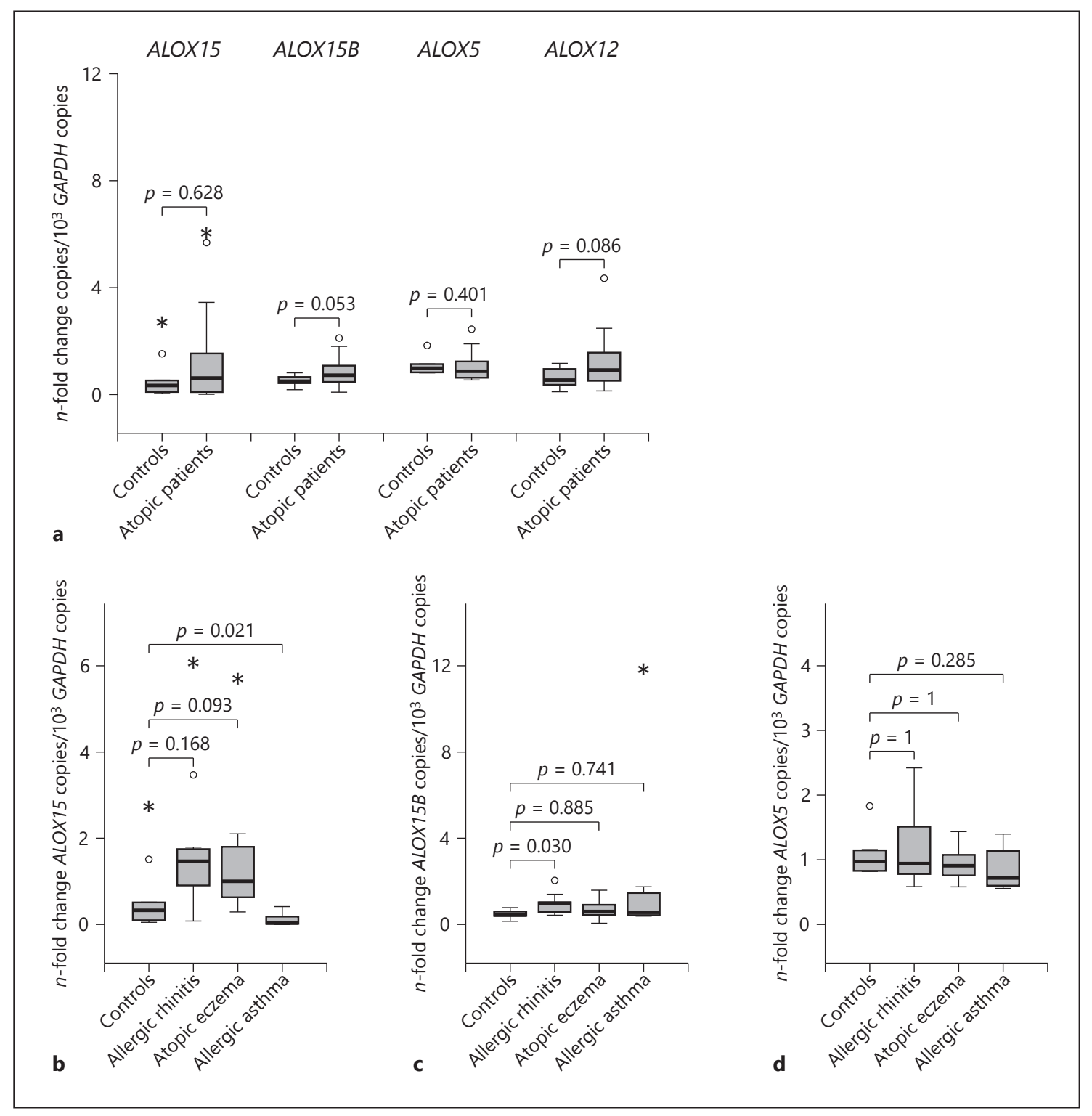

Fig. 2. Expression of ALOX isoforms in blood leukocytes of healthy human volunteers and patients suffering from atopic diseases. Expression of ALOX isoforms was determined by qRT-PCR, and the ratio of mRNA copies of the target gene per 1,000 copies of GAP$D H$ mRNA was calculated. a Comparison of the expression of different ALOX-isoforms in blood leukocytes of healthy controls $(n=9)$ and pooled atopic patients $(n=34)$ independently of their pathological entities. b-d Comparison of the expression of

fold increase in ALOX15 expression in allergic rhinitis (Fig. 2b). For atopic eczema patients, a similar 2.23-fold increase in ALOX15 expression was observed (Fig. 2b), but here again only borderline significance was calculated $(p=0.093)$. For atopic asthma patients a significant ( $p=0.021)$ decrease in ALOX15 expression was ob-
ALOX15 (b), ALOX15B (c) and ALOX5 (d) in blood leukocytes of healthy controls $(n=9)$ and patients suffering from different entities of allergic diseases (allergic rhinitis $n=11$, atopic eczema $n=$ 11 , atopic asthma $n=12$ ). Statistical evaluation was carried out applying the nonparametric Mann-Whitney $U$ test with subsequent Bonferroni adjustment; $p<0.05$ was considered as statistically significant.

served (Fig. 2b). For ALOX15B no significant alterations were observed for atopic eczema and atopic asthma patients ( $p=0.885$ and 0.930 , respectively). However, we found a small ( 1.93 -fold) but significant $(p=0.030)$ increase in $A L O X 15 B$ expression in allergic rhinitis patients (Fig. 2c). 


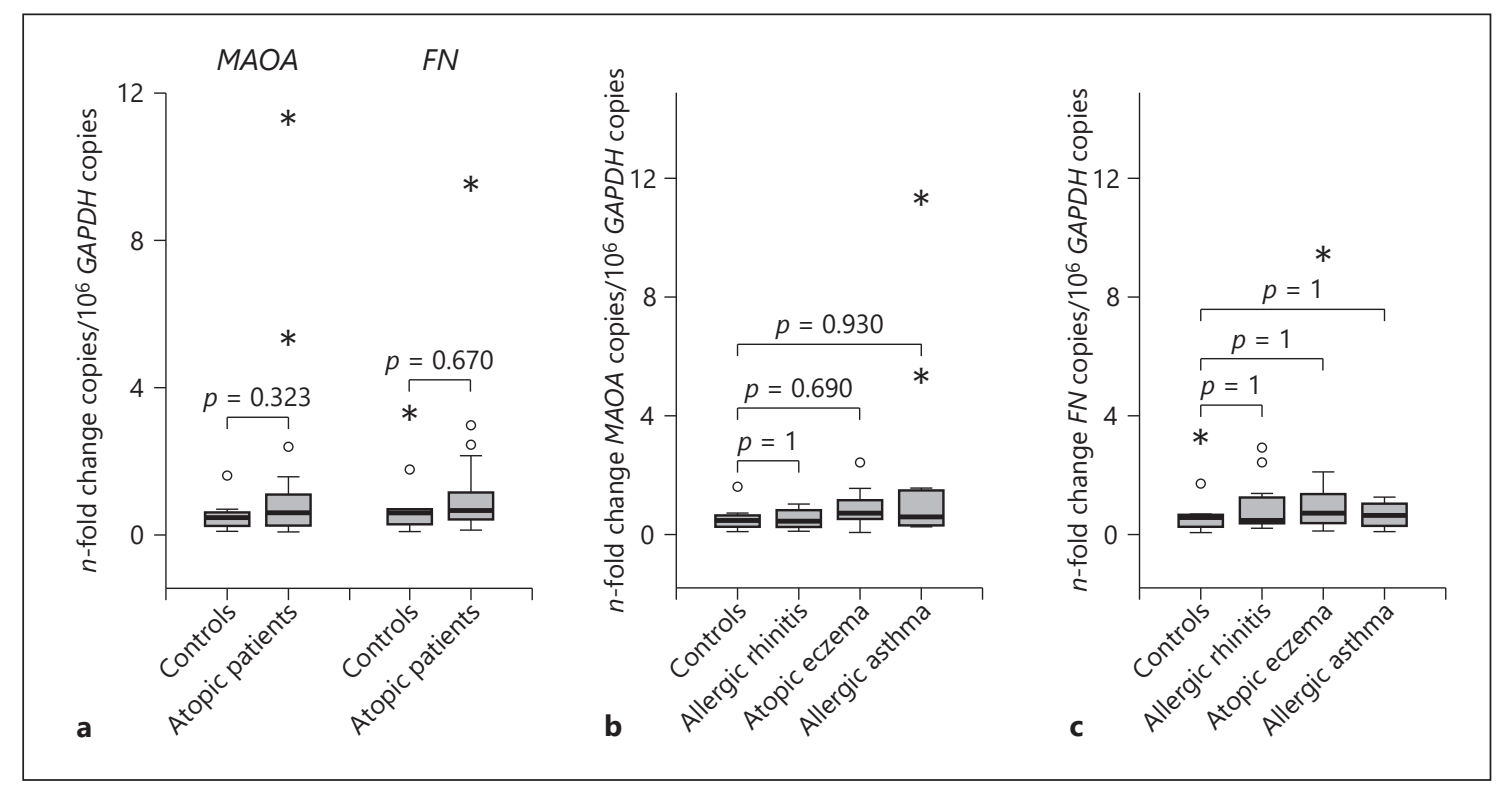

Fig. 3. Expression of additional IL-4-sensitive genes (MAOA, FN) in blood leukocytes of healthy human volunteers and patients suffering from atopic diseases. Expression of IL-4-sensitive genes was quantified by qRT-PCR, and the ratio of $\mathrm{mRNA}$ copies of the target gene per $1 \times 10^{6}$ copies of GAPDH mRNA was calculated. a Comparison of the expression $M A O A$ and $F N$ in blood leukocytes of healthy controls $(n=9)$ and pooled atopic patients $(n=34)$ inde-

ALOX5 and its major pro-inflammatory reaction product leukotriene $\mathrm{B}_{4}$ has been implicated in the pathogenesis of bronchial asthma and other inflammatory diseases, including skin inflammation [7, 23-25]. Moreover, an ALOX5 inhibitory activity has been shown for antiinflammatory medicinal plants, and pharmaceutical preparations of these plants have been used for the treatment of inflammatory skin diseases in traditional medicine [26]. These data suggested a role of ALOX5 in atopic diseases and thus, we quantified expression of ALOX5 in atopic asthma patients but did not observe significant differences to healthy controls (Fig. 2d). This was neither the case for other atopic entities (Fig. 2d). These results are consistent with data obtained in a previous report [27].

In addition to $A L O X 15$ and $A L O X 15 B$, expression of $F N$ and of MAOA is also upregulated in vitro by IL-4 [17]. When we explored whether such expression regulations can also be observed in vivo, we did not find any differences between atopic patients and healthy controls (Fig. 3a). We neither observed significant differences for MAOA (Fig. 3b) and FN (Fig. 3c) expression when the different atopic entities were compared separately. pendently of their pathological entities. b, c Comparison of the expression of MAOA (b) and $F N(\mathbf{c})$ in blood leukocytes of healthy controls $(n=9)$ and patients suffering from different entities of allergic diseases (allergic rhinitis $n=11$, atopic eczema $n=11$, atopic asthma $n=12$ ). Statistical evaluation was carried out applying the nonparametric Mann-Whitney $U$ test with subsequent Bonferroni adjustment.

Table 1. Correlation analyses

\begin{tabular}{|c|c|c|c|c|}
\hline \multirow[t]{2}{*}{ Correlated variables } & \multicolumn{2}{|c|}{$\begin{array}{l}\text { Atopic patients + } \\
\text { healthy controls }\end{array}$} & \multicolumn{2}{|c|}{$\begin{array}{l}\text { Atopic patients } \\
\text { only }\end{array}$} \\
\hline & $r$ & $p$ & $r$ & $p$ \\
\hline IgE vs. IL-4 & 0.351 & 0.072 & 0.131 & 1 \\
\hline IL-4 vs. $A L O X 15$ & -0.270 & 0.160 & -0.352 & 0.082 \\
\hline IL- 4 vs. $A L O X 15 B$ & 0.036 & 1 & -0.083 & 1 \\
\hline$A L O X 15$ vs. $A L O X 5$ & 0.371 & 0.028 & 0.430 & 0.022 \\
\hline IL-4 vs. $M A O A$ & -0.144 & 0.716 & -0.173 & 0.654 \\
\hline IL-4 vs. fibronectin & 0.253 & 0.204 & 0.204 & 0.496 \\
\hline$M A O A$ vs. $A L O X 15$ & -0.177 & 0.510 & -0.292 & 0.188 \\
\hline Fibronectin vs. $A L O X 15$ & 0.192 & 0.436 & 0.228 & 0.392 \\
\hline
\end{tabular}

Significant results are italicized. Correlation analyses were carried out with the IBM SPSS Statistics software packages; the 2 -tailed Spearman $\rho$ test was employed.

\section{Correlation Analysis}

When we correlated plasma IgE and plasma IL-4 levels, we did not find a significant correlation regardless of whether we only included the data from atopic patients or from atopic patients and healthy controls (Table 1). 


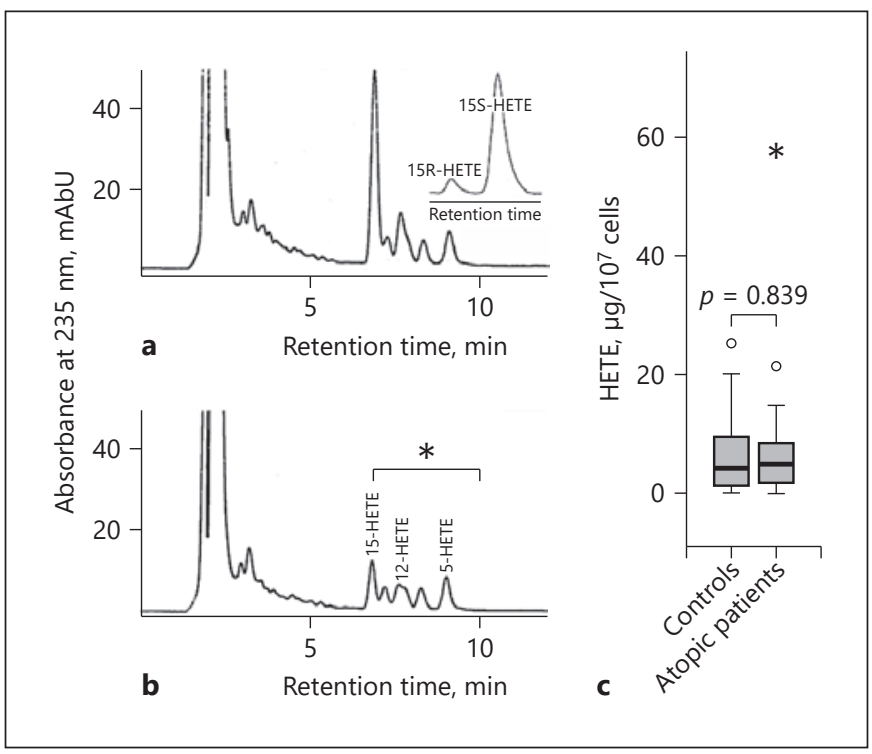

Fig. 4. In vitro and ex vivo ALOX activity assays employing isolated monocytes as enzyme source. a RP-HPLC analysis of arachidonic acid oxygenation products formed during the in vitro incubation of human peripheral monocytes with arachidonic acid. Monocytes cultured for $72 \mathrm{~h}$ in the presence of $25 \mathrm{ng} / \mathrm{mL}$ human recombinant IL-4. Inset Enantiomer analysis of 15-HETE isolated by RP-HPLC. $\mathbf{b}$ RP-HPLC analysis of arachidonic acid oxygenation products formed during the in vitro incubation of human peripheral monocytes with arachidonic acid. Monocytes were cultured for $72 \mathrm{~h}$ in the absence of IL-4. The arachidonic acid oxygenation products were identified according to retention time of authentic standards. ${ }^{*}$ Chromatographic region integrated for quantification and statistical evaluation. c Statistical evaluation of HETE formation by blood monocytes prepared from healthy volunteers $(n=17)$ and patients suffering from atopic diseases $(n=21)$ applying the nonparametric Mann-Whitney $\mathrm{U}$ test.

Similarly, we did not observe a correlation between the plasma IL-4 levels and ALOX15 and ALOX15B expression (Table 1). However, we observed a significant positive correlation between the expression of ALOX15 and ALOX5 (Table 1). When we correlated the IL-4 plasma levels with $M A O A$ or $F N$ expression, we did not find significant correlations, and this was also the case when we compared the expression of these two genes with that of ALOX15 (Table 1).

\section{Fatty Acid Oxygenase Activity of Blood Monocytes}

When human peripheral monocytes were cultured in vitro for $72 \mathrm{~h}$ in the presence of IL-4 $(25 \mathrm{ng} / \mathrm{mL})$, they exhibit a strong arachidonic acid oxygenase activity as indicated by HPLC analysis (Fig. 4a). In fact, the major oxygenation product co-chromatographed in RP-HPLC with an authentic standard of 15-HETE (hydroxyeicosatetraenoic acid). Chiral-phase HPLC (inset) indicated the strong preponderance of 15S-HETE indicating its enzymatic origin. When the monocytes were cultured in the absence of IL-4, an unspecific product pattern was detected (Fig. 4b) suggesting that ALOX15 and $A L$ $O X 15 B$ are not expressed. This conclusion is consistent with qRT-PCR data for ALOX 15 mRNA ( 0.25 copies of ALOX 15 mRNA $/ 10^{3}$ GAPDH copies in the absence of IL- 4 vs. 554.67 copies of $A L O X 15$ mRNA/ $10^{3}$ GAPDH copies in its presence).

When we isolated monocytes from atopic patients and assayed their fatty acid oxygenase activity, we found that the corresponding chromatograms were similar to those of corresponding control cells (comparable with Fig. 4b). For statistical evaluation we quantified the peak areas of the HETE region (from 6 to $10 \mathrm{~min}$ ) but did not detect any significant difference between monocytes prepared from healthy controls and atopic patients (Fig. 4c).

\section{Discussion}

Role of IL-4 and ALOX Isoforms in Atopic Diseases

ALOX isoforms have been implicated in the pathogenesis of atopic diseases [28] and cysteinyl leukotriene receptor antagonists (montelukast, zafirlukast) [29], and leukotriene biosynthesis inhibitors (zileuton) [30] are currently used for the treatment of bronchial asthma and allergic eye diseases. Moreover, the anti-inflammatory activity of some medicinal plants has been related to their inhibitory activity of the ALOX5 pathway, and pharmaceutical extracts of such plants have been employed for the treatment of atopic eczema [26]. Moreover, a number of ALOX inhibitors exhibit antioxidative properties, and some of them are useful chemopreventive agents of oxidative stress $[31,32]$. However, the precise roles of pro-inflammatory leukotrienes [32] and anti-inflammatory lipoxins/resolvins $[33,34]$ in atopic diseases are not completely understood. A recent study reported that patients suffering from atopic dermatitis show an altered profile of lipids in blood plasma [35] and in the skin [36] and these data have been suggested to point towards an increased production of ALOX-derived pro-inflammatory mediator [35]. IL-4 has previously been identified as regulator of the ALOX pathway in monocytes/macrophages and dendritic cells [21, 22, 37], and microarray-based expression profiles suggested that IL-4 strongly upregulates the expression of 
ALOX15 and ALOX15B [21, 22, 37, 38]. Unfortunately, the in vivo relevance of this in vitro effect has not been tested so far. Since atopic patients have frequently increased IL-4 plasma concentrations $[11,17]$, we hypothesized increased expression levels of IL-4-sensitive genes in peripheral leukocytes of such patients. Although we found significantly increased IgE plasma levels in allergic patients (Fig. 1a), systemic IL-4 concentration levels were only significantly augmented in allergic asthma patients (Fig. 1b, d), and there was no significant correlation between the individual $\operatorname{IgE}$ and IL-4 levels. Analyzing the expression of IL-4-sensitive genes (ALOX15, ALOX15B, ALOX5, FN, MAOA) in blood leukocytes, we did not find significant differences between atopic patients and healthy controls (Fig. 2a and 3a). Thus, the increased circulating IL-4 levels, which are observed in some atopic entities, do not significantly upregulate the expression of IL-4-sensitive genes.

\section{Circulating IL-4 Levels Are Not High Enough to}

\section{Induce Expression of IL-4-Sensitive Genes}

To explore the possible reasons for this in vitro versus in vivo discrepancy, we compared the IL-4 concentrations used for induction of the in vitro effect with the elevated IL-4 concentrations reached in atopic disease. The circulating IL-4 levels in atopic patients varied between 0.7 and $2.4 \mathrm{pg} / \mathrm{mL}$ plasma but in the literature there is a higher degree of variation $(1-500 \mathrm{pg} / \mathrm{mL})[11$, 16-19]. To induce in vitro expression of IL-4-sensitive genes in monocytes, IL- 4 concentrations between 10,000 and $50,000 \mathrm{pg} / \mathrm{mL}$ were usually employed $[21,22,38]$. For other cell types (epithelial cells [39], endothelial cells [40], fibroblasts [41], preadipocytes [42]), IL-4 concentrations as low as $200 \mathrm{pg} / \mathrm{mL}$ were used. Thus, the IL-4 concentrations employed for the in vitro experiments with isolated monocytes were considerably higher than the in vivo concentrations detected in atopic patients.

\section{Possible Impact of Medication on IL-4-Induced}

\section{Expression of IL-4-Sensitive Genes}

Some patients enrolled in this study have previously received different types of antiatopic medication (online suppl. Table S1), and due to ethical reasons treatment was not interrupted. A limitation of this study is that we did not test whether the medication might have inhibited expression of IL-4-sensitive genes. Indeed, IL-4-dependent induction of $A L O X 15$ expression in monocytes was partly inhibited by high $(100 \mathrm{nM})$ concentrations of hydrocor- tisone [22]. However, hydrocortisone was not systemically applied. Thus, the possibility that antiatopic medication might have interfered with the expression of IL-4-sensitive genes is unlikely.

\section{Expression of ALOX5 in Blood Leukocytes of Atopic Patients}

Leukotrienes have been implicated in atopic diseases $[30,43]$ and ALOX5 functions as key enzyme in leukotriene biosynthesis [20]. In previous reports conflicting data have been published on the expression of ALOX5 in peripheral leukocytes in atopic patients. In a primary study no significant elevation of $A L O X 5$ expression has been detected in asthmatic patients [27]. In a later trial a small ( 2 -fold) but significant $(p<0.05)$ upregulation of ALOX5 expression in blood leukocytes of asthmatic patients was observed [44]. Our data are consistent with the original report, and insignificant differences were obtained for all atopic entities (Fig. 2d). The reasons for these conflicting results are unclear but in addition to the different inclusion criteria [27, 44], methodological differences might contribute.

\section{Acknowledgment}

This work was supported by a research grant of the Deutsche Forschungsgemeinschaft - DFG (Ku961/11-1).

\section{Statement of Ethics}

The study was approved by the Ethic Commission of the Charité - Universitätsmedizin Berlin (EA1/052/16). All patients provided written informed consent.

\section{Disclosure Statement}

The authors declare that they do not have any conflicts of interest with the content of this article.

\section{Author Contributions}

E.B.-M. carried out RNA preparations and qRT-PCR. A.K. performed preparation of peripheral monocytes, LOX activity assays and HPLC analyses. D.H. designed the PCR primers and prepared the external amplification standards. E.M.-B. and S.St. performed quantification of the circulating IL-4 levels. E.B.-M., H.K., J.W.F. and T.Z. designed the study. H.K. and E.M.-B. evaluated the experimental data and drafted the manuscript. All co-authors were involved in working out the final version of the paper. 


\section{References}

1 Halken S, Larenas-Linnemann D, Roberts G, Calderón MA, Angier E, Pfaar O, et al. EAACI guidelines on allergen immunotherapy: prevention of allergy. Pediatr Allergy Immunol. 2017 Dec;28(8):728-45.

2 Henriksen L, Simonsen J, Haerskjold A, Linder M, Kieler H, Thomsen SF, Stensballe LG. Incidence rates of atopic dermatitis, asthma, and allergic rhinoconjunctivitis in Danish and Swedish children. J Allergy Clin Immunol. 2015;136:360-6. e362.

3 Zuberbier T, Lötvall J, Simoens S, Subramanian SV, Church MK. Economic burden of inadequate management of allergic diseases in the European Union: a GA(2) LEN review. Allergy. 2014 Oct;69(10):1275-9.

4 Farzanfar D, Dowlati Y, French LE, Lowes MA, Alavi A. Inflammation: A Contributor to Depressive Comorbidity in Inflammatory Skin Disease. Skin Pharmacol Physiol. 2018; 31(5):246-51.

5 Yanagibashi T, Satoh M, Nagai Y, Koike M, Takatsu K. Allergic diseases: from bench to clinic - contribution of the discovery of interleukin-5. Cytokine. 2017 Oct; 98:59-70.

6 Müller-Decker K, Furstenberger G, Neumann M, Schnolzer M. Differential protein expression in the epidermis of wild-type and COX-2 transgenic mice. Skin Pharmacol Physiol. 2006;19(2):89-94.

7 Hanssen SC, Hendriks AG, Keijsers RM, van Erp PE, van der Vleuten CJ, Seyger $\mathrm{MM}$, et al. Response of the endothelium to the epicutaneous application of of leukotriene B4. Skin Pharmacol Physiol. 2017;30(6): 306-14.

8 Fanning LB, Boyce JA. Lipid mediators and allergic diseases. Ann Allergy Asthma Immunol. 2013 Sep;111(3):155-62

9 Umar M, Sastry KS, Al Ali F, Al-Khulaifi M, Wang E, Chouchane AI. Vitamin D and the Pathophysiology of Inflammatory Skin Diseases. Skin Pharmacol Physiol. 2018;31(2): 74-86.

10 Siracusa MC, Kim BS, Spergel JM, Artis D. Basophils and allergic inflammation. J Allergy Clin Immunol. 2013 Oct;132(4):789-801.

11 Tavakkol Afshari J, Farid Hosseini R, Hosseini Farahabadi S, Heydarian F, Boskabady $\mathrm{MH}$, Khoshnavaz R, et al. Association of the expression of IL-4 and IL-13 genes, IL-4 and IgE serum levels with allergic asthma. Iran J Allergy Asthma Immunol. 2007 Jun;6(2):6772.

12 Hou DD, Di ZH, Qi RQ, Wang HX, Zheng S, Hong YX, et al. Sea Buckthorn (Hippophaë rhamnoides L.) Oil Improves Atopic Dermatitis-Like Skin Lesions via Inhibition of NF$\kappa \mathrm{B}$ and STAT1 Activation. Skin Pharmacol Physiol. 2017;30(5):268-76.

13 Wright JD, Chu HM, Huang $\mathrm{CH}$, Ma C, Chang TW, Lim C. Structural and Physical Basis for Anti-IgE Therapy. Sci Rep. 2015 Jun; 5(1):11581
14 Wynn TA. Type 2 cytokines: mechanisms and therapeutic strategies. Nat Rev Immunol. 2015 May; 15(5):271-82.

15 Kleiner G, Marcuzzi A, Zanin V, Monasta L, Zauli G. Cytokine levels in the serum of healthy subjects. Mediators Inflamm. 2013; 2013:434010.

16 Antczak A, Domańska-Senderowska D, GórskiP,Pastuszak-LewandoskaD,NielepkowiczGoździńska A, Szewczyk K, et al. Analysis of changes in expression of IL-4/IL-13/STAT6 pathway and correlation with the selected clinical parameters in patients with atopic asthma. Int J Immunopathol Pharmacol. 2016 Jun;29(2):195-204.

17 Shang H, Cao XL, Wan YJ, Meng J, Guo LH. IL-4 gene polymorphism may contribute to an increased risk of atopic dermatitis in children. Dis Markers. 2016;2016:1021942.

18 Gharaee H, Shayegan MR, Khakzad MR, Kianoush S, Varasteh AR, Sankian M, et al. The expression of vascular endothelial growth factor in pterygium tissue of atopic patients. Int Ophthalmol. 2014 Dec;34(6):1175-81.

19 Hussein YM, Shalaby SM, Nassar A, Alzahrani SS, Alharbi AS, Nouh M. Association between genes encoding components of the IL-4/IL-4 receptor pathway and dermatitis in children. Gene. 2014 Jul;545(2):276-81.

20 Chaitidis P, O’Donnell V, Kuban RJ, Bermudez-Fajardo A, Ungethuem U, Kühn H. Gene expression alterations of human peripheral blood monocytes induced by medium-term treatment with the $\mathrm{TH} 2$-cytokines interleukin-4 and -13. Cytokine. 2005 Jun;30(6):36677.

21 Wuest SJ, Crucet M, Gemperle C, Loretz C, Hersberger M. Expression and regulation of 12/15-lipoxygenases in human primary macrophages. Atherosclerosis. 2012 Nov;225(1): 121-7.

22 Conrad DJ, Kuhn H, Mulkins M, Highland E, Sigal E. Specific inflammatory cytokines regulate the expression of human monocyte 15-lipoxygenase. Proc Natl Acad Sci USA. 1992 Jan;89(1):217-21.

23 Rådmark O, Werz O, Steinhilber D, Samuelsson B. 5-Lipoxygenase, a key enzyme for leukotriene biosynthesis in health and disease. Biochim Biophys Acta. 2015 Apr;1851(4): 331-9.

24 Haeggström JZ, Funk CD. Lipoxygenase and leukotriene pathways: biochemistry, biology, and roles in disease. Chem Rev. 2011 Oct; 111(10):5866-98.

25 Konya V, Mjösberg J. Lipid mediators as regulators of human ILC2 function in allergic diseases. Immunol Lett. $2016 \mathrm{Nov}$; 179:36-42.

26 Frum Y, Viljoen AM. In vitro 5-lipoxygenase and anti-oxidant activities of South African medicinal plants commonly used topically for skin diseases. Skin Pharmacol Physiol. 2006; 19(6):329-35

27 Kuitert LM, Newton R, Barnes NC, Adcock IM, Barnes PJ. Eicosanoid mediator expres- sion in mononuclear and polymorphonuclear cells in normal subjects and patients with atopic asthma and cystic fibrosis. Thorax. 1996 Dec;51(12):1223-8.

28 Kuhn H, Banthiya S, van Leyen K. Mammalian lipoxygenases and their biological relevance. Biochim Biophys Acta. 2015 Apr; 1851(4):308-30.

29 Zhang HP, Jia CE, Lv Y, Gibson PG, Wang G. Montelukast for prevention and treatment of asthma exacerbations in adults: systematic review and meta-analysis. Allergy Asthma Proc. 2014;35:278-287.

30 Berger W, De Chandt MT, Cairns CB. Zileuton: clinical implications of 5-Lipoxygenase inhibition in severe airway disease. Int J Clin Pract. 2007 Apr;61(4):663-76.

31 Yang S, Zhou B, Xu W, Xue F, Nisar MF, Bian C, et al. Nrf2- and Bach1 May Play a Role in the Modulation of Ultraviolet A-Induced Oxidative Stress by Acetyl-11-Keto- $\beta$-Boswellic Acid in Skin Keratinocytes. Skin Pharmacol Physiol. 2017;30(1):13-23.

32 Drazen JM. Anti-leukotrienes as novel antiinflammatory treatments in asthma. Adv Exp Med Biol. 2002;507:217-21.

33 Serhan CN, Chiang N. Resolution phase lipid mediators of inflammation: agonists of resolution. Curr Opin Pharmacol. 2013 Aug; 13(4):632-40.

34 Bäck M, Powell WS, Dahlén SE, Drazen JM, Evans JF, Serhan CN, et al. Update on leukotriene, lipoxin and oxoeicosanoid receptors: IUPHAR Review 7. Br J Pharmacol. 2014 Aug;171(15):3551-74.

35 Mihály J, Marosvölgyi T, Szegedi A, Köröskényi $K$, Lucas $R$, Törőcsik $D$, et al. Increased FADS2-derived n-6 PUFAs and reduced n-3 PUFAs in plasma of atopic dermatitis patients. Skin Pharmacol Physiol. 2014;27(5): 242-8.

36 Tessema EN, Gebre-Mariam T, Neubert RH, Wohlrab J. Potential Applications of PhytoDerived Ceramides in Improving Epidermal Barrier Function. Skin Pharmacol Physiol. 2017;30(3):115-38

37 Bhattacharjee A, Shukla M, Yakubenko VP, Mulya A, Kundu S, Cathcart MK. IL-4 and IL13 employ discrete signaling pathways for target gene expression in alternatively activated monocytes/macrophages. Free Radic Biol Med. 2013 Jan;54:1-16.

38 Kuhn H, Gehring T, Schröter A, Heydeck D. Cytokine-dependent expression regulation of ALOX15. J Cytokine Biol. 2016;1(2):106-19.

39 Brinckmann R, Topp MS, Zalán I, Heydeck D, Ludwig P, Kühn H, et al. Regulation of 15-lipoxygenase expression in lung epithelial cells by interleukin-4. Biochem J. 1996 Aug;318(Pt 1):305-12.

40 Lee YW, Kühn H, Kaiser S, Hennig B, Daugherty $\mathrm{A}$, Toborek $\mathrm{M}$. Interleukin 4 induces transcription of the 15-lipoxygenase I gene in human endothelial cells. J Lipid Res. 2001 May;42(5):783-91. 
41 Chen B, Tsui S, Boeglin WE, Douglas RS, Brash AR, Smith TJ. Interleukin-4 induces 15-lipoxygenase-1 expression in human orbital fibroblasts from patients with Graves disease. Evidence for anatomic site-selective actions of Th2 cytokines. J Biol Chem. 2006 Jul;281(27):18296-306.
42 Tsao CH, Shiau MY, Chuang PH, Chang YH, Hwang J. Interleukin-4 regulates lipid metabolism by inhibiting adipogenesis and promoting lipolysis. J Lipid Res. 2014 Mar;55(3):38597.

43 Genovese L, Corbo A, Sibilla S. An Insight into the Changes in Skin Texture and Properties following Dietary Intervention with a Nutricosmeceutical Containing a Blend of Collagen Bioactive Peptides and Antioxidants. Skin Pharmacol Physiol. 2017;30(3):146-58.
44 Koshino T, Takano S, Houjo T, Sano Y, Kudo $\mathrm{K}$, Kihara H, et al. Expression of 5-lipoxygenase and 5-lipoxygenase-activating protein mRNAs in the peripheral blood leukocytes of asthmatics. Biochem Biophys Res Commun. 1998 Jun;247(2):510-3. 